\title{
Application in the Construction of Teaching Resources Database Data Mining
}

\author{
Bo Lin \\ Information Engineering Department \\ Tianjin Bohai Vocational Technology College \\ TianJin, P.R.China \\ linboei@163.com
}

\author{
Yanping Chen \\ Information Engineering Department \\ Tianjin Bohai Vocational Technology College \\ TianJin, P.R.China \\ chenyanpingei@163.com
}

\begin{abstract}
s-with the development of teaching resources storehouse construction projects continue to enhance, how to solve the problem of benign growth system of teaching resources has appeared. This paper discusses the application of data mining technology in the construction of teaching resources database.
\end{abstract}

Keywords- teaching resource library.

\section{FOREWORD}

Teaching resources storehouse construction in recent years the school has become an important direction of education informatization. However, how to evaluate the real-time applicability, the teaching resources database has been established, how to evaluate the resource value through the behavior analysis on user access, which is also an important aspect of teaching resources database development. Data mining technology and the rapid development in order to solve the problem brought effective methods and means.

\section{THE DATA MINING TECHNOLOGY OVERVIEW}

\section{A. Introduction to data mining}

Data mining (Data Mining) from a mass of data, extracting potential, valuable knowledge (model or rules) process, which belongs to a deep level data analysis method. It is an interdisciplinary subject, including machine learning, statistics, neural network, database, pattern recognition, rough set, fuzzy mathematics and other related technology. Data mining technology consists of three main parts: the algorithm and technology; data; modeling capabilities. It combines many areas of theory and technology, can extract hidden information or pattern, and has potential application value. Data mining is an effective tool for dealing with massive data, many fields both at home and abroad has been widely used, and has achieved good results, but in the field of education, its application research is still in its early stage. Data mining process is as follows:

1) determine the mining purposes

Clearly define the business problem, clear the purpose of data mining is an important step in data mining.

2) the data preparation

a) Data selection:

Search all related to business objects to internal and external data, and choose the suitable for data mining application data.

b) Data preprocessing:
Quality research data, preparing for the further analysis. And determine the type will be carried out mining operations.

c) Data conversion:

convert the data into a model. This analysis model is established for the mining algorithm. To create a truly suitable for data mining analysis model is the key to successful data mining.

3) data mining

The obtained through the conversion of data mining. In addition to improving the mining algorithm to choose the appropriate outside, the rest all work can be done automatically.

4) analysis and assimilation

a) Results:

Interpretation and evaluation results, the analytical methods used should be as general data mining operations and decide, usually requires the use of visualization technology.

b) The assimilation of knowledge:

the analysis of knowledge integration and organizational structure of business information system.

\section{B. The data mining algorithm description}

Processing algorithm: first, breadth first search the itemset space, starting from $\mathrm{k}=0$, produced for the length of candidate item set $\mathrm{Ck}+1 \mathrm{k}+1$. Candidate itemsets of all its subsets are frequent item sets. C1 by the current set of all items in the form, in the $\mathrm{k}$ layer generates all length $\mathrm{k}+1$ item set.

It consists of two steps: the first step, the same layer set Fk self connection. The Fk has the same (k-1) - itemsets prefix connection for the growth of the candidate item set K. The second step is to prune, if all the length set to a subset of the $\mathrm{K}$ in $\mathrm{Fk}$, the set can be used as candidate items have been added to $\mathrm{Ck}+1$.

In order to calculate the all length $\mathrm{k}$ candidate itemsets in the database support, the level of representation, need to scan the database again. During each scan, the database of each transaction records, which contains all the candidate ksupport of itemset count plus 1 . All frequent itemsets have been added to Fk k-. This process until $\mathrm{Ck}+1$ is equal to the end. 


\section{APPLICATION OF DATA MINING TECHNOLOGY IN THE NETWORK TEACHING RESOURCES IN THE LIBRARY}

The system of teaching resources at present in China has gradually developed, however, is still at the primary stage in how to promote healthy growth, teaching resource library how to dynamically evaluate the knowledge system, the main problem is reflected in the:

A. Dynamic interactive function is not strong. The system of teaching resources are piling up teaching material, the user can only passively browsing content, not actually involved in the system of discovery, creation of more active learning.

B. The system of intelligent low. Can not provide the interface of intelligent, mobilize the interest of the user.

C. Knowledge evaluation function of the system is not strong. Not according to the user's evaluation of the dynamic remodeling knowledge system.

\section{TO EXPLORE THE APPLICATION OF DATA MINING TECHNOLOGY IN THE CONSTRUCTION OF TEACHING RESOURCES DATABASE.}

Network information based on data mining is an important application of data mining. Network data mining from the network related resources and behaviors from useful pattern and hidden information process. General as shown below using the algorithms:

Network analysis provides in-depth analysis of data, detailed teaching resource library operation. Using these data, we can solve the following problems:
A. Continuous improvement of teaching resource database, the purpose is more steady, improve user satisfaction. Through mining the user learning behavior, find user browsing patterns, and further optimization of teaching resource library organization structure, the information that the user wants to faster, more efficient way to show out, to meet the user access habits.

B. View and analyze resource flow pattern teaching. Find the user's needs and interests, the user is devoted to the optimization of the access to large quantities of Webpage, using server stored in advance to speed up the browsing speed, improve the user experience.

C. Provide personalized teaching resources storehouse. According to different users, their learning interest through data mining, and provide different learning content and mode, really do have a definite object in view, teach students in accordance with their aptitude, to achieve the purpose of personalized teaching resources library. Provide valuable information for teachers. Such as through the students' papers are analyzed, the relationship between the subject and the other useful hidden information, used to improve the test structure, difficulty coefficient correction.

D. By mining the various save teaching resources on learning record information, combined with the courseware knowledge base, automatic re combination of course content, the teaching rules.

\section{THE CONCLUSION}

As the project construction of college teaching resource library to increase the amount of information, network teaching resources will increase sharply, and the needs of the user's access to teaching resources development plays a key role. Data mining technology will be widely used in the construction of network teaching resource library.

\section{REFERENCES}

[1] Agrawal R, Gollapudi S, Kannan A, et al. Data Mining for Improving Textbooks. ACM SIGKDD Explorations, 2011, 13(2): 7-19.

[2] Calders T, Pechenizkiy M. Introduction to the Special Section on Educational Data Mining. ACM SIGKDD Explorations, 2011, 13(2): 3-6.

[3] Desmarais M.C. Mapping Question Items to Skills with Non-negative Matrix Factorization. ACM SIGKDD Explorations, 2011, 13(2): 30-36.

[4] Pardos Z.A, Baker R.S.J.d, Gowda S.M, et al. The Sum is Greater than the Parts: Ensembling Models of Student Knowledge in Educational Software, 2011, 13(2): 37-44.

[5] Language can be restrained. The application of data mining technology in the information technology teaching evaluation. China extramural education.2011,1. 